\title{
A Randomized Pilot Study of the Effect of Trelagliptin and Alogliptin on Glycemic Variability in Patients with Type 2 Diabetes
}

\author{
Rimei Nishimura · Takeshi Osonoi · Yasuhiro Koike · Kouji Miyata (D) \\ Yukio Shimasaki
}

Received: August 9, 2019 / Published online: September 27, 2019

(C) The Author(s) 2019

\section{ABSTRACT}

Introduction: This open-label, parallel-group, exploratory study examined the effects of two dipeptidyl peptidase 4 (DPP4) inhibitors on glycemic variability (GV) in patients with type 2 diabetes.

Methods: Randomized patients with glycated hemoglobin A1c of at least $6.5 \%$ to less than $8.5 \%$ received trelagliptin $100 \mathrm{mg}(n=13)$ once weekly or alogliptin $25 \mathrm{mg}(n=14)$ once daily for 29 days. Continuous glucose monitoring was performed before the start of the treatment period (baseline) and from day 21 to 29 , inclusive. The primary endpoint was change from baseline in the standard deviation (SD) of 24-h blood glucose values, measured daily for 7 days

Enhanced Digital Features To view enhanced digital features for this article go to https://doi.org/10.6084/ m9.figshare.9805409.

R. Nishimura

Department of Diabetes, Metabolism and

Endocrinology, Jikei University School of Medicine,

Minato-ku, Tokyo, Japan

T. Osonoi

Department of Internal Medicine, Nakakinen

Clinic, Naka-city, Ibaraki, Japan

Y. Koike · K. Miyata $(\bowtie) \cdot$ Y. Shimasaki

Japan Medical Affairs, Japan Pharma Business Unit,

Takeda Pharmaceutical Company Limited,

Chuo-ku, Tokyo, Japan

e-mail: kouji.miyata@takeda.com (day 22-28) of the treatment period. Secondary and additional efficacy endpoints included changes in glycemic parameters and the rate of DPP4 inhibition, respectively. Adverse events (AEs) were monitored to assess safety.

Results: Mean change from baseline in the SD of 24 -h blood glucose (95\% confidence interval) at day 28 was $-7.35(-15.13,0.44)$ for trelagliptin and $-11.63(-18.67,-4.59)$ for alogliptin. In both treatment groups, glycemic parameters improved and the rate of DPP4 inhibition was maintained. Three patients reported AEs; no severe treatment-emergent AEs were reported in either group.

Conclusion: Once-weekly trelagliptin and once-daily alogliptin improved glycemic control and reduced GV without inducing hypoglycemia.

Trial Registration: ClinicalTrials.gov (NCT027 71093) and JAPIC (JapicCTI-163250).

Funding: Takeda Pharmaceutical Company, Ltd.

Keywords: Alogliptin; Continuous glucose monitoring; Dipeptidyl peptidase 4 inhibitors; Efficacy; Glycemic variability; Hypoglycemia; Safety; Trelagliptin; Type 2 diabetes

\section{INTRODUCTION}

The worldwide prevalence of diabetes mellitus (DM) in adults was estimated to be $8.4 \%$ in 2017 
and was predicted to rise to $9.9 \%$ in 2045 [1], which is similar to the estimated rise in prevalence in Japan (7.7\% in 2017 to $8.3 \%$ in 2045) [2]. The primary goal of diabetes treatment is to achieve optimal glycemic control [3]. Glycemic control is currently assessed using the most common marker, glycated hemoglobin A1c (HbA1c), which closely correlates with the average plasma glucose level over a preceding 8to 12 -week period [4], as well as fasting blood glucose in a clinical setting. Although HbA1c provides a reliable measure of long-term glycemic control and chronic hyperglycemia [5], there is evidence to suggest that glycemic variability (GV) may contribute independently to diabetes-related complications [3, 6-9]. GV measured by standard deviation (SD) reflects blood glucose oscillations that occur throughout the day, including hypoglycemic and postprandial increases, and variability between daily means that are unlikely to be captured by HbA1c or fasting blood glucose measurements $[3,10]$.

Evidence from some studies suggests that, similar to chronic hyperglycemia [11], longterm high GV may increase the risk of microvascular and macrovascular complications associated with type 2 DM (T2DM) $[8,9]$, while severe hypoglycemia is associated with increased mortality $[6,7]$. Although the precise mechanism by which GV may adversely affect cardiovascular risk is not fully understood, some evidence suggests that acute GV increases oxidative stress [12] and endothelial dysfunction [13], both of which may contribute to the development of cardiovascular complications. GV has also been strongly associated with increased incidence of hypoglycemia [10], as well as contributing to increased mean blood glucose values [3].

Dipeptidyl peptidase 4 (DPP4) inhibitors are antidiabetic agents that bind to the enzyme DPP4 to inhibit the degradation of the incretins, glucagon-like peptide 1 (GLP-1), and glucosedependent insulinotropic polypeptide (GIP), both of which play key roles in glucose homeostasis and glycemic control [14]. By prolonging the activity of endogenous GLP-1 and GIP, DPP4 inhibitors can improve postprandial hyperglycemia, without inducing hypoglycemia, in patients with T2DM [15]. Trelagliptin is an oral antidiabetic medication that inhibits DPP4 and improves glycemic control in patients with diabetes [16]. Unlike early DPP4 inhibitors, which are administered once or twice daily, trelagliptin is administered once weekly [17]. However, despite the less frequent dosing, a phase 3 clinical study in patients with T2DM demonstrated that the efficacy of once-weekly trelagliptin was non-inferior to that of oncedaily alogliptin in lowering HbA1c [18]. Although various oral hypoglycemic agents have been evaluated in Japan using the Guideline for Clinical Evaluation of Oral Hypoglycemic Agents [19], the effects of these agents, including alogliptin and trelagliptin, on GV have not been investigated. Therefore, the present exploratory pilot study was undertaken to investigate the effect of trelagliptin $100 \mathrm{mg}$ once weekly and alogliptin $25 \mathrm{mg}$ once daily on GV in patients with T2DM.

\section{METHODS}

\section{Study Design}

This was a randomized, open-label, parallelgroup, exploratory study that evaluated the effect of trelagliptin $100 \mathrm{mg}$ administered once weekly or alogliptin $25 \mathrm{mg}$ administered once daily for 4 weeks. GV was assessed in patients with T2DM using continuous glucose monitoring (CGM) (Fig. 1). An assigned person generated the allocation list. Allocation was conducted at the enrollment center using a web case enrollment system on day 1. Eligible patients were randomized 1:1 using the allocation list to receive either trelagliptin $100 \mathrm{mg}$ or alogliptin $25 \mathrm{mg}$, stratified by baseline HbA1c (less than $7.5 \%$ or at least $7.5 \%$ ) and age (less than 65 or at least 65 years). Randomization was preceded by a 2-day observation period, followed by a 29 -day treatment period. All procedures performed in studies involving human participants were in accordance with the ethical standards of the institutional and/or national research committee and with the 1964 Helsinki declaration and its later amendments or comparable ethical standards. Informed consent was 


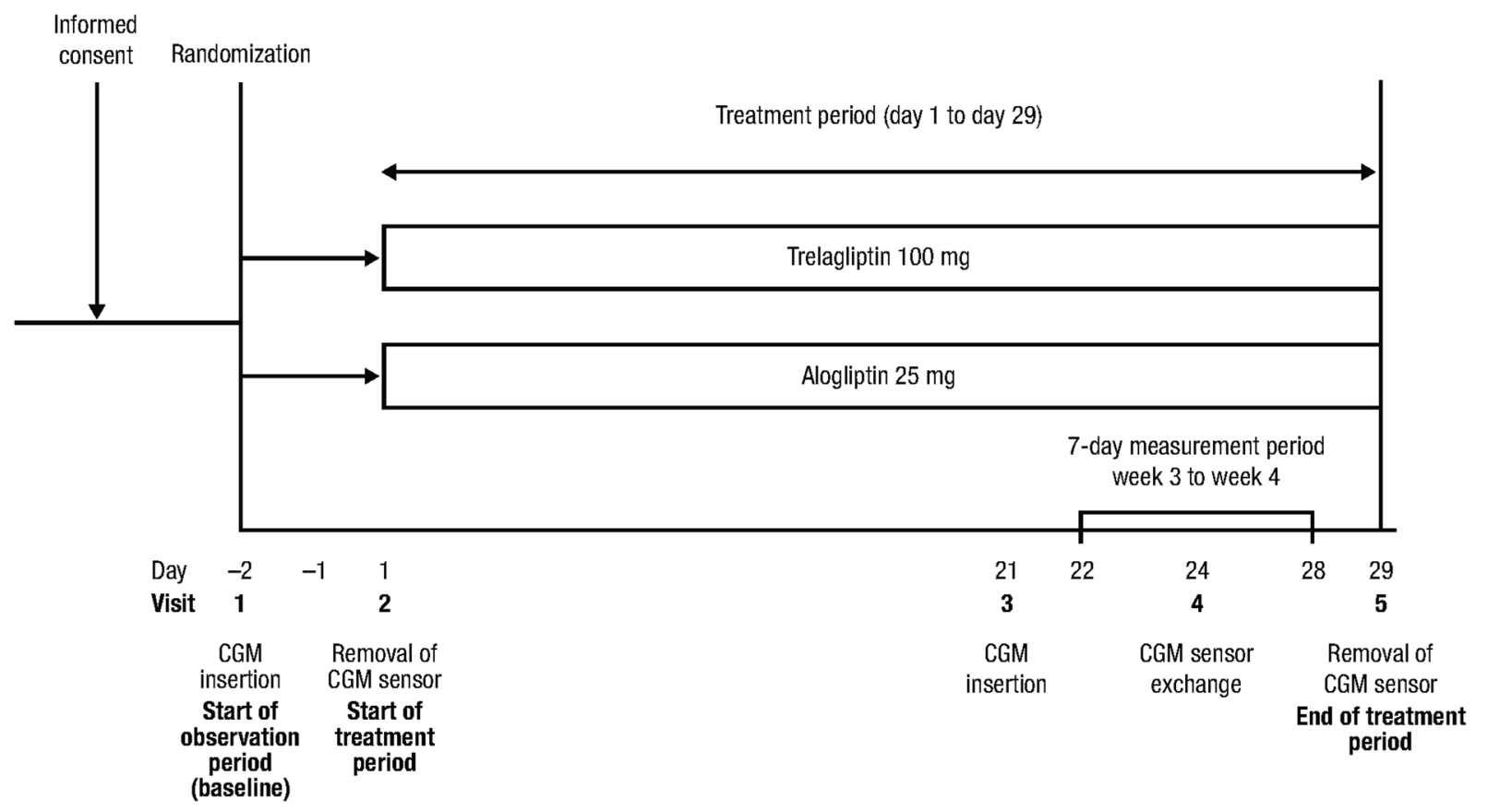

Fig. 1 Study design. CGM continuous glucose monitoring

obtained from all individual participants included in the study. The study protocol, informed consent form, and other regulation-specified documents were reviewed and approved by the Independent Ethics Committees at Nakakinen Clinic. The study was conducted between June 2016 and April 2017 at two study sites (medical institutions) in Japan, and is registered at ClinicalTrials.gov (NCT02771093) and JAPIC (JapicCTI-163250). The study was registered on May 11, 2016.

\section{Participants}

Patient eligibility criteria included patients aged at least 20 years old with T2DM, HbA1c (National Glycohemoglobin Standardization Program value) of at least $6.5 \%$ and less than $8.5 \%$, and stable glycemic control with at most $1 \%$ change in HbA1c at the start of the observation period [day -2 (visit 1)] vs the preceding 6 weeks. Patients must have been receiving stable diet and exercise therapy (if required) for at least 4 weeks before the start of the observation period (day - 2). Key exclusion criteria included patients who were receiving antidiabetic medications within 4 weeks of the start of the observation period, or patients who changed (including discontinuation or interruption) 3-hydroxy-3-methylglutaryl-coenzyme A (HMG-CoA) reductase inhibitors or received new HMG-CoA reductase inhibitors within 4 weeks before the start of the observation period. Concomitant use of medications that may affect efficacy evaluation was prohibited throughout the duration of the study; these included antidiabetic medications other than the allocated study drugs, systemic glucocorticoids (excluding local/topical preparations), estrogen preparations, HMG-CoA reductase inhibitors other than those used at the time of informed consent, and acetaminophen.

During CGM, patients presented photographs of their meals to allow the investigator to monitor patient compliance to diet therapy.

\section{CGM}

CGM was conducted using Medtronic iPro ${ }^{\circledR} 2$ (Medtronic MiniMed, Inc. Northridge, CA, USA), and was performed for 2 days during the observation period and 9 days (day 21-29) 
during the treatment period (Fig. 1). Patients calibrated their CGM during the observation and treatment periods by measuring their daily blood glucose levels at a minimum of three time points on the first day of the CGM (at least $2 \mathrm{~h}$ after the recorder was connected, $2 \mathrm{~h}$ after the first measurement, and at bedtime) and at four time points from the second day onward (before breakfast, before lunch, before the evening meal, and at bedtime).

\section{Study Endpoints and Assessments}

The primary endpoint was change from baseline ( $4 \mathrm{~h}$ before breakfast to $20 \mathrm{~h}$ post-breakfast on day -1$)$ in the SD of 24-h blood glucose values $(\mathrm{mg} / \mathrm{dL})$ measured each day from day 22 to 28 of the treatment period. Secondary efficacy endpoints included change from baseline in 24-h mean blood glucose on each day during day 22-28 of the treatment period; area under the glucose concentration-time curve (AUC) during periods when blood glucose was at least $180 \mathrm{mg} / \mathrm{dL}$; change in 3-h postprandial blood glucose over time, $3 \mathrm{~h}$ after breakfast, lunch, and evening meal; change in AUC over time during periods when blood glucose levels were less than $70 \mathrm{mg} / \mathrm{dL}$; changes in mean amplitude glycemic excursions (MAGE) from baseline; change from baseline in mean 24-h, daytime, and nocturnal blood glucose by visit; and AUC of blood glucose and change from baseline by visit.

Additional efficacy endpoints included change from baseline (day - 2) in plasma DPP4 activity; plasma DPP4 inhibition rate; change from baseline in glycoalbumin; change from baseline in 1,5-anhydroglucitol (AG); fasting blood glucose; fasting glucagon; fasting GLP-1; and fasting GIP at visit 3 and visit 5 of the 9-day CGM period (day 1 and day 29 of the treatment period).

The safety endpoints were the frequency and nature of treatment-emergent adverse events (TEAEs) over the 29-day treatment period.

\section{Statistical Analyses}

The planned sample size of 30 patients was determined with consideration of the feasibility of performing CGM in numerous patients, and was not based on a statistical power calculation.

The full analysis set (FAS), which was defined as all patients who were randomized and received at least one dose of the study drug, was used for the efficacy analyses unless otherwise specified. The safety analysis set included all patients who received at least one dose of the study drug.

For efficacy endpoints, summary statistics, including point-estimate and two-sided 95\% confidence interval (CI) for the mean for each group, were calculated. Mean change (95\% CI) of SD of 24-h blood glucose from baseline each day from day 22 to 28 (vs the start of the observation period) were calculated to evaluate changes in the SD of 24-h blood glucose values.

A TEAE was defined as an adverse event (AE) with a date of onset on or after the first dose of the study drug. AEs were coded using the Japanese version of the Medical Dictionary for Regulatory Activities, version 20.0, and displayed using System Organ Class and Preferred Term.

\section{RESULTS}

A total of 27 patients signed the informed consent form, met the eligibility criteria for randomization ( $n=13$, once-weekly trelagliptin $100 \mathrm{mg} ; n=14$, once-daily alogliptin $25 \mathrm{mg}$ ), and completed the study treatment (Fig. 2).

The baseline demographics and patient characteristics are described in Table 1. A total of $53.8 \%$ and $71.4 \%$ of patients in the trelagliptin group and alogliptin group were male, respectively. The mean duration of T2DM was 5.62 years and 6.15 years in the trelagliptin group and alogliptin group, respectively.

All patients in both treatment groups fully complied (at least 75\%) with the study medication. 


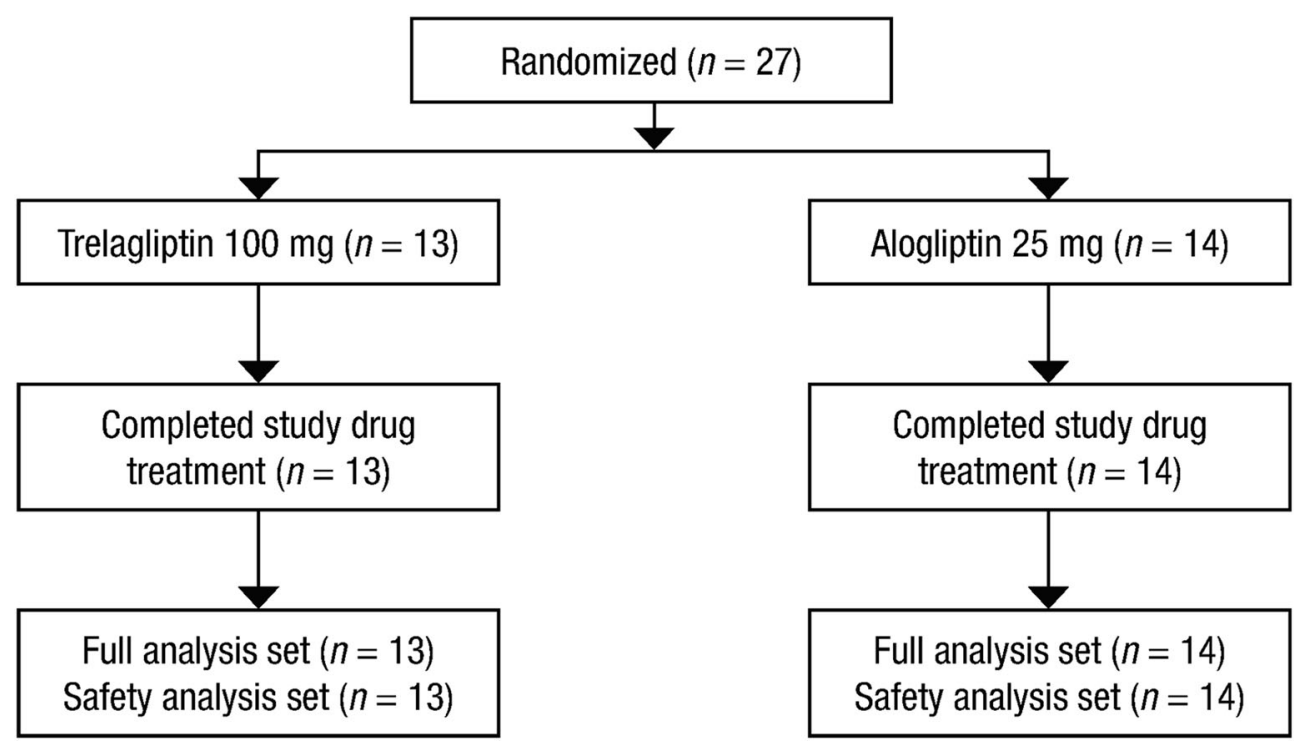

Fig. 2 Schematic representation of patient disposition

Table 1 Baseline characteristics of the randomized population

\begin{tabular}{|c|c|c|}
\hline Characteristic & $\begin{array}{l}\text { Trelagliptin } \\
100 \mathbf{~ m g} \\
(n=13)\end{array}$ & $\begin{array}{l}\text { Alogliptin } \\
25 \mathrm{mg} \\
(n=14)\end{array}$ \\
\hline $\begin{array}{l}\text { Age, years, mean } \\
\text { (SD) }\end{array}$ & $62.8(10.14)$ & $62.7(7.64)$ \\
\hline Male, $n(\%)$ & $7(53.8)$ & $10(71.4)$ \\
\hline $\begin{array}{l}\text { Height, cm, mean } \\
\text { (SD) }\end{array}$ & $160.9(10.40)$ & $164.4(7.17)$ \\
\hline $\begin{array}{l}\text { Weight, kg, mean } \\
\text { (SD) }\end{array}$ & $66.45(14.11)$ & $64.15(9.59)$ \\
\hline $\begin{array}{l}\text { BMI, } \mathrm{kg} / \mathrm{m}^{2} \text {, mean } \\
\quad(\mathrm{SD})\end{array}$ & $25.45(2.95)$ & $23.65(2.40)$ \\
\hline $\begin{array}{l}\text { Duration of } \mathrm{DM}, \\
\text { years, mean }(\mathrm{SD})\end{array}$ & $5.62(4.47)$ & $6.15(4.68)$ \\
\hline $\begin{array}{l}\text { HbAlc (NGSP), \%, } \\
\text { mean (SD) }\end{array}$ & $7.29(0.62)$ & $7.19(0.34)$ \\
\hline
\end{tabular}

$B M I$ body-mass index, $D M$ diabetes mellitus, $H b A I c$ glycated hemoglobin A1c, NGSP National Glycohemoglobin Standardization Program, $S D$ standard deviation

\section{Mean SD of 24-h Blood Glucose (Primary Efficacy Endpoint)}

Mean SD (observed value) of 24-h blood glucose at baseline was 38.18 in the trelagliptin group and 40.44 in the alogliptin group. In the trelagliptin group, the mean SD (observed values) was 30.68 on day 22 and 30.84 on day 28. In the alogliptin group, mean SD (observed values) was 27.41 on day 22 and 28.81 on day 28 .

Figure 3 shows the mean change from baseline in the SD of 24-h blood glucose at day 22 and day 28 , which was -7.51 (95\% CI - 17.10, $2.08)$ and -7.35 (95\% CI $-15.13,0.44)$, respectively, in the trelagliptin group, and - 13.04 (95\% CI - 20.84, - 5.23) and - 11.63 (95\% CI $-18.67,-4.59)$, respectively, in the alogliptin group.

\section{Secondary Efficacy Endpoints}

Changes from baseline for secondary efficacy endpoints are shown in Table 2. The observed values for all secondary efficacy glycemic parameters at day 22 and day 28 numerically decreased from baseline in both groups, with the exception of mean nocturnal blood glucose, which increased from baseline at day 28 in the trelagliptin group. Of note, mean AUC over 


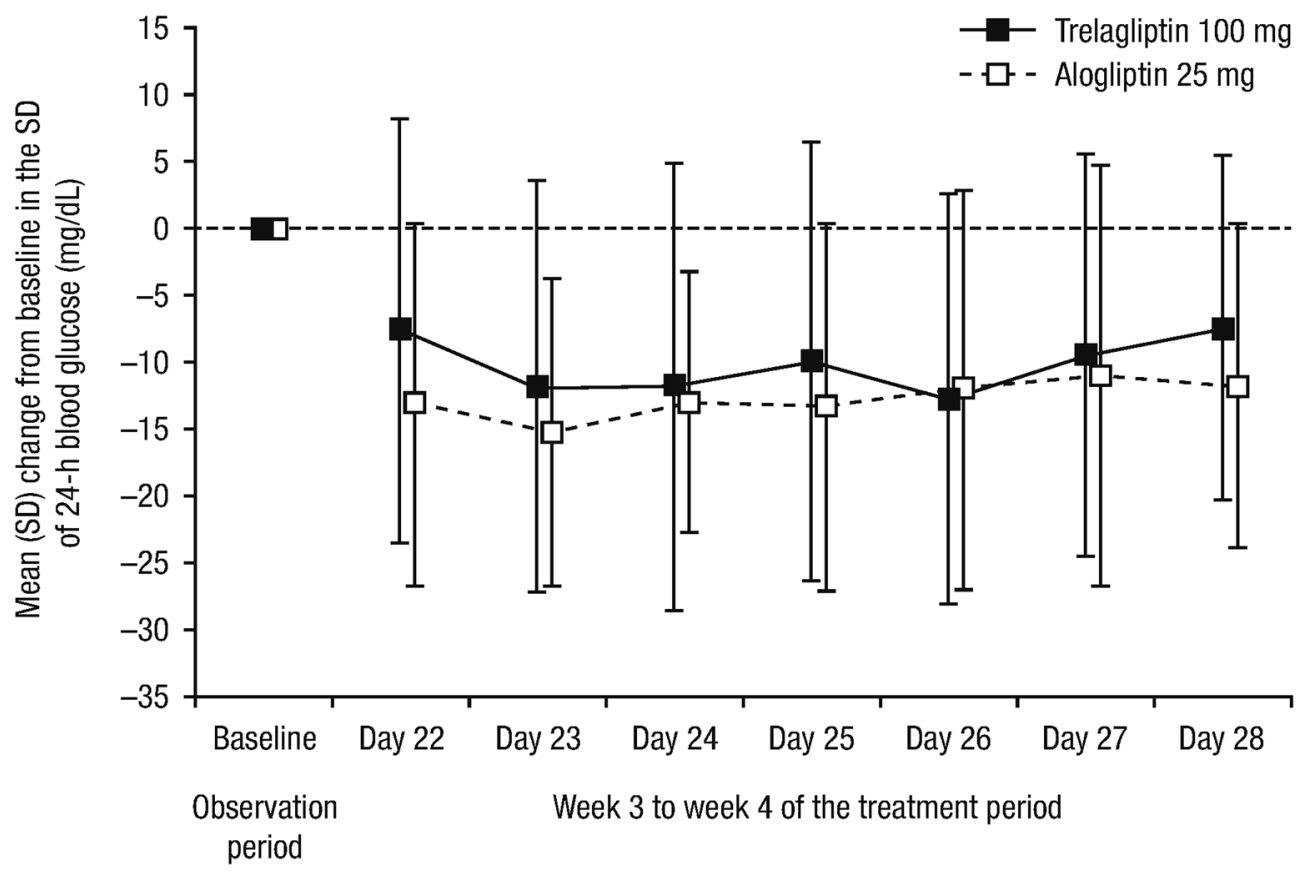

Fig. 3 Change from baseline of SD of 24-h blood glucose. SD standard deviation

time at blood glucose less than $70 \mathrm{mg} / \mathrm{dL}$ (hypoglycemia) at baseline was $43.0 \mathrm{mg} / \mathrm{dL} \mathrm{min}$ in the trelagliptin group and $0.0 \mathrm{mg} / \mathrm{dL}$ min in the alogliptin group. Mean change of AUC from baseline was $-31.2 \mathrm{mg} / \mathrm{dL}$ min $\quad(95 \% \quad$ CI $-105.8,43.3)$ in the trelagliptin group and $+0.4 \mathrm{mg} / \mathrm{dL}$ min in the alogliptin group at day 22, and $-43.0 \mathrm{mg} / \mathrm{dL} \min$ (95\% CI - 136.3, 50.3 ) in the trelagliptin group and $0.0 \mathrm{mg} /$ $\mathrm{dL}$ min in the alogliptin group at day 28.

\section{Additional Efficacy Endpoints}

Changes in other glycemic parameters from baseline to day 21 and day 29 are shown in Table 3. Plasma DPP4 inhibition rate at day 21 and day 29 are shown in Fig. 4. A reduction in glycoalbumin, fasting blood glucose, fasting glucagon, and plasma DPP4 activity, and an increase in the observed values of 1,5-AG, fasting GLP-1, and fasting GIP were seen at day 21 and day 29 in both groups compared with baseline (Table 3 ).

The frequency distributions of glucose values obtained from CGM appeared to shift toward lower glucose values from day 22 to day 28 in both groups, compared with baseline (Fig. 5).

\section{Safety}

One patient (7.7\%) receiving once-weekly trelagliptin and two patients (14.3\%) receiving once-daily alogliptin experienced TEAEs. One patient in the trelagliptin group experienced dizziness. In the alogliptin group, one patient experienced gastric mucosal lesion, feeling abnormal, and viral upper respiratory tract infection, and one patient experienced eczema. All TEAEs were mild in intensity, and no drugrelated TEAEs were reported. No deaths, no serious TEAEs, or no serious TEAEs leading to drug discontinuation were reported in the study.

\section{DISCUSSION}

$\mathrm{GV}$ is an important physiological phenomenon in patients with T2DM, which contributes not only to increased mean blood glucose values but also to the development of complications in chronic diabetes [3]. There is evidence to 


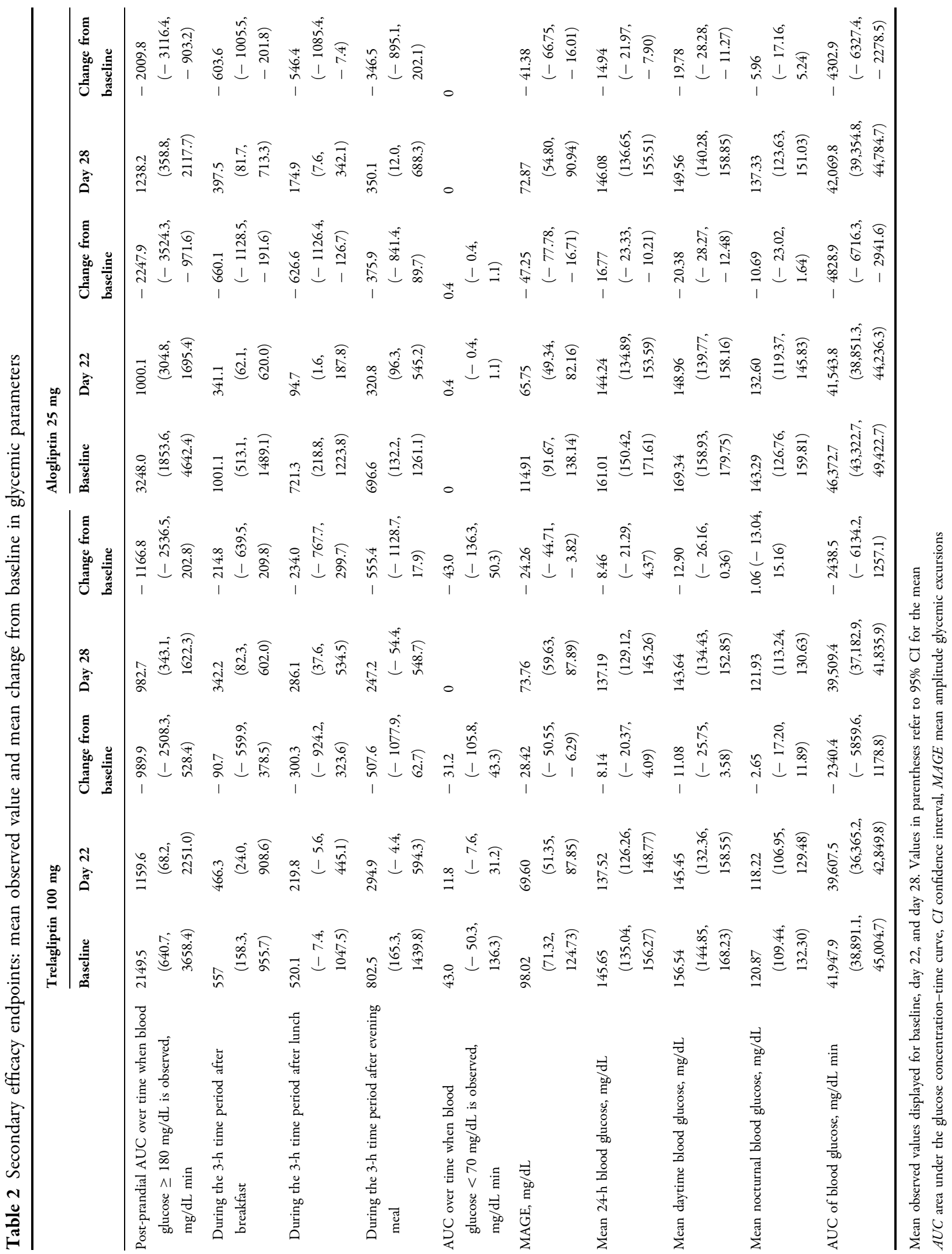




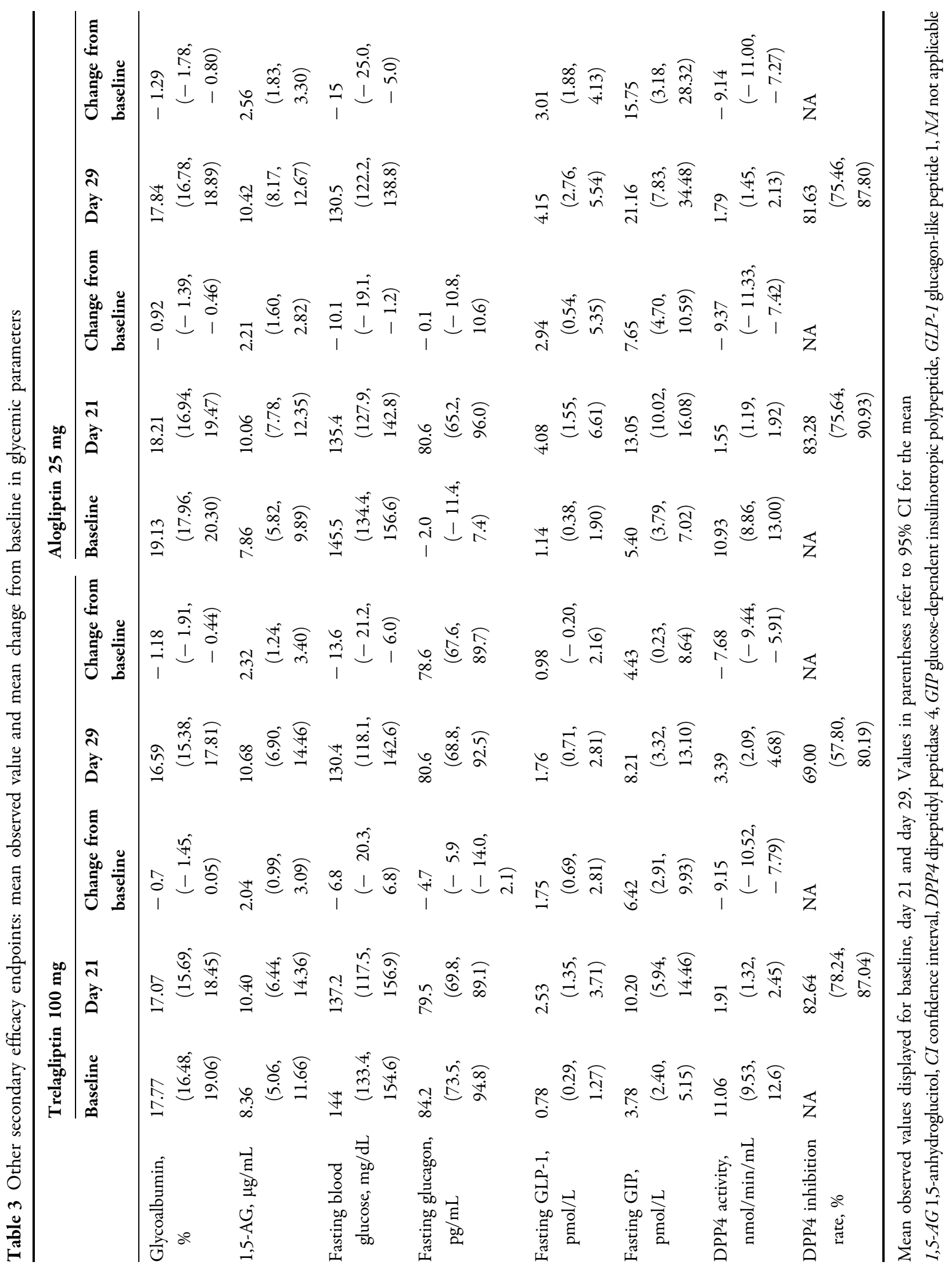




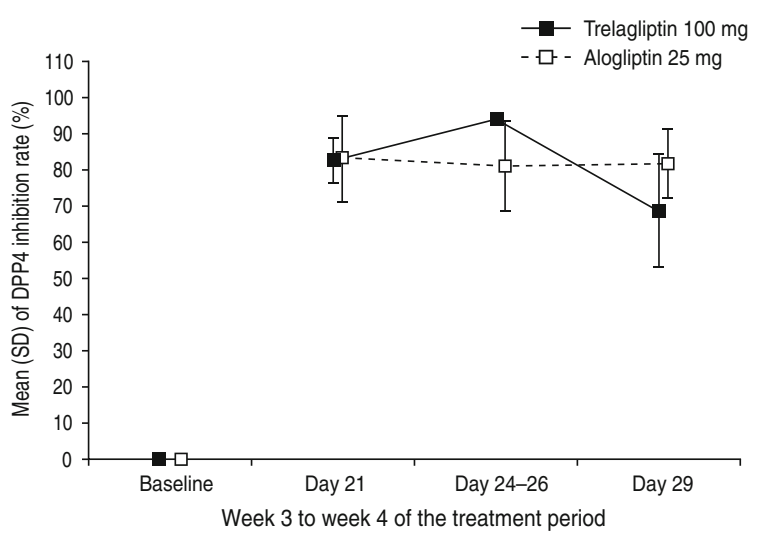

Fig. 4 Inhibition rate of plasma DPP4. DPP4 dipeptidyl peptidase 4, SD standard deviation

suggest that tighter HbA1c control alone is insufficient for preventing diabetes complications, in particular cardiovascular events caused by long-term high GV $[20,21]$. GV is therefore an important consideration in the management of blood glucose [22]. It has been reported that DPP4 inhibitors have the potential to improve glycemic control and to reduce glucose fluctuations, by increasing active serum GLP-1 and GIP concentrations via glucose-dependent insulin secretion $[23,24]$. Indeed, the long-term effect of once-daily alogliptin on glycemic control in Japanese patients with T2DM has been investigated, demonstrating a significant reduction in HbA1c $(p=0.0001)$ over 3.5 years [25]. Trelagliptin administered once weekly is also efficacious [18], is well-tolerated as a longterm mono- and combination therapy treatment option [26], and is non-inferior to oncedaily alogliptin [18]. However, the effect of oral hypoglycemic agents, including once-weekly trelagliptin $100 \mathrm{mg}$ and once-daily alogliptin $25 \mathrm{mg}$, on GV in patients with T2DM has not been investigated previously with regard to the frequency of administration.

In the present study, the administration of trelagliptin $100 \mathrm{mg}$ once weekly or alogliptin $25 \mathrm{mg}$ once daily decreased blood glucose levels and reduced GV to a similar extent in patients with T2DM, as measured by changes in SD of 24-h blood glucose and MAGE. Notably, this effect was maintained for 1 week after trelagliptin administration. During periods of high blood glucose (at least $180 \mathrm{mg} / \mathrm{dL}$ ), postprandial and 24-h data obtained by CGM showed that both trelagliptin and alogliptin suppressed hyperglycemia. Again, this effect was maintained for up to 8 days after administration of once-weekly trelagliptin. We also quantitatively demonstrated that the risk of hypoglycemia was low in patients treated with trelagliptin or alogliptin, which is consistent with a previous report of DPP4 inhibitors and hypoglycemia $[15,27]$.

Inhibition of plasma DPP4 activity was maintained from day 21 to 29 , with a mean DPP4 inhibition rate at day 21 and day 29 of $82.64 \%$ and $69.00 \%$, respectively, in the trelagliptin group, and $83.28 \%$ and $81.63 \%$, respectively, in the alogliptin group. Although the day 29 DPP4 inhibition rate was numerically lower with trelagliptin than with alogliptin, it should be noted that the rate with trelagliptin was measured 9 days after the final dose; this was comparable to the result of a phase 2 study that reported a DPP4 inhibition rate of $77.4 \%$ with once-weekly trelagliptin, 7 days after the final dose [28]. Overall, these data support the current dosing frequencies of trelagliptin once weekly and alogliptin once daily.

By inhibiting DPP4 activity, trelagliptin and alogliptin increase plasma levels of the intact, active forms of GLP-1 and GIP. The glucose-dependent insulinotropic effects of both GLP-1 and GIP, as well as the glucagonostatic effect of GLP-1, are thought to underlie the therapeutic efficacy of trelagliptin and alogliptin. In the present study, a decrease in 3-h postprandial AUC after each meal was observed at day 22 and day 29 compared with baseline, and AUC at blood glucose levels less than $70 \mathrm{mg} / \mathrm{dL}$ was 0 at day 29 in both groups. These results indicate glucose-dependent insulinotropic effects of trelagliptin and alogliptin, and support their effects on reducing blood glucose without inducing hypoglycemia. Currently, it is unknown whether DPP4 inhibitory activity measured in the blood is similar to that in cells within the intestinal tract and periphery of the pancreas, and this warrants further investigation.

Both trelagliptin $100 \mathrm{mg}$ and alogliptin $25 \mathrm{mg}$ have been shown previously to have favorable safety profiles $[18,26,28]$ and, 

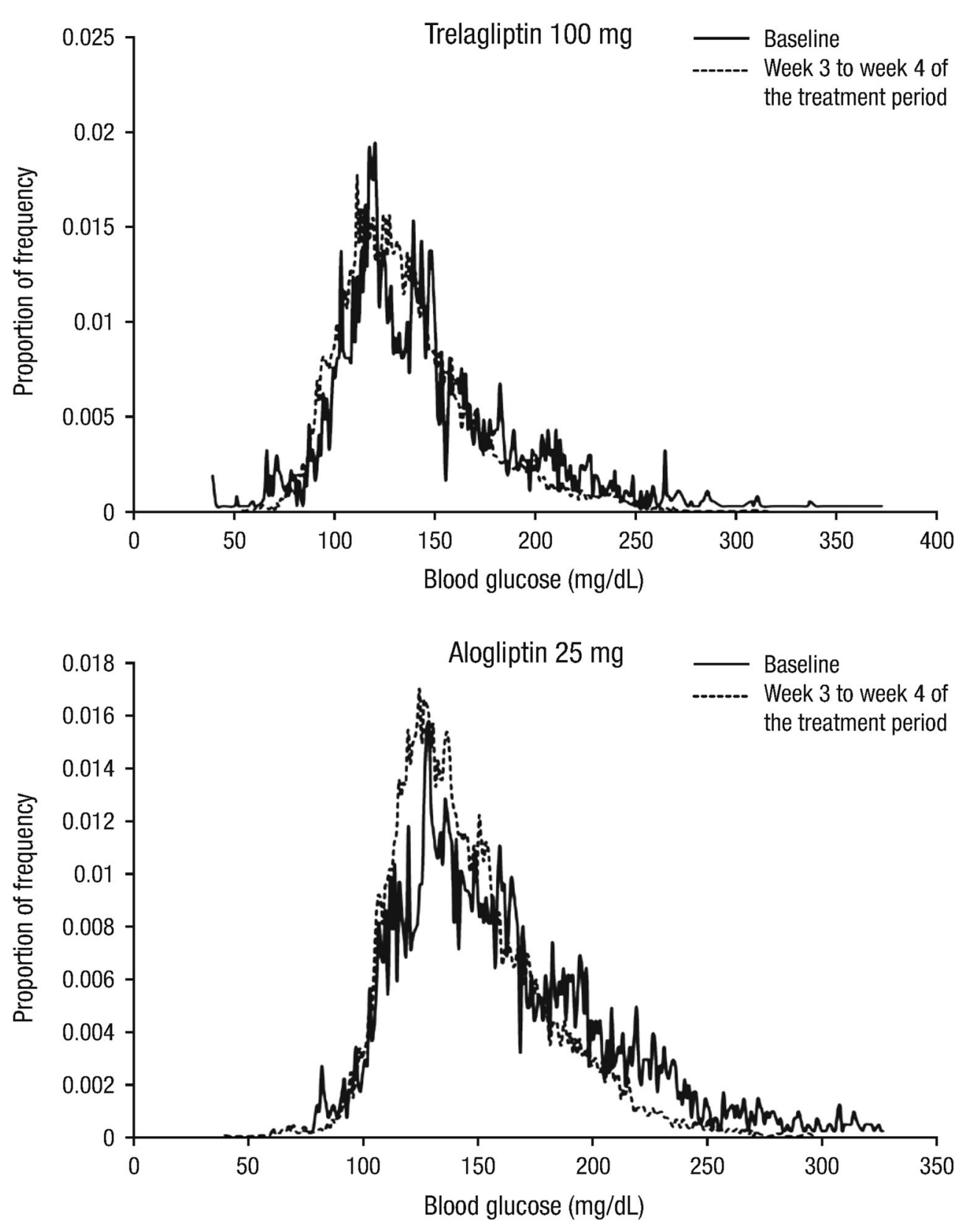

Fig. 5 Frequency distributions of glucose values obtained from CGM. CGM continuous glucose monitoring

importantly, no new safety signals were identified in this study. Of note, Inagaki et al. reported that switching from once-daily sitagliptin to once-weekly trelagliptin in patients with T2DM and stable glycemic control in combination with diet and exercise therapy did not result in major changes in HbA1c and fasting plasma glucose [29]. Safety and tolerability profiles remained favorable and there were no deaths, serious adverse events, or cases of hypoglycemia [29]. The selection between trelagliptin and a once-daily DPP4 inhibitor will depend on the treatment policy of the physician, quality of life of the patient, and the patient's lifestyle and treatment preferences. For patients continuing long-term treatment, a once-weekly DPP4 inhibitor may be preferable for reducing the number of treatment visits, while delivering a stable and 
prolonged glucose-lowering effect that is comparable to that of a once-daily DPP4 inhibitor.

It has been suggested that DPP4 inhibitors may have a protective effect on pancreatic beta-cells by elongating the telomere length [30]. It has also been reported that enhancement of the GLP-1 receptor signaling enhances beta-cell function, proliferation, and regeneration, and diminishes beta-cell apoptosis [31]. Such protection may be continued and reinforced with long-term continued use of trelagliptin or alogliptin.

This study has several limitations. One limitation was the small number of enrolled patients. This was mainly due to the challenges of obtaining informed consent from patients with T2DM, based on the study design using CGM. CGM has been available through health insurance in Japan since 2009; however, patients are sometimes hesitant to use it because of the stress caused by insertion and removal of the CGM sensor, as well as the restriction of patients' activities during monitoring [32]. CGM at baseline was therefore of a short duration to avoid inconvenience to the patients. An additional limitation could be the imbalance in CGM glucose values between treatment groups at baseline, although eligible patients were randomized 1:1 to receive either trelagliptin $100 \mathrm{mg}$ or alogliptin $25 \mathrm{mg}$ stratified by baseline HbA1c and age. Further studies enrolling larger patient populations are warranted. In addition, further investigation of the mode of action of onceweekly and once-daily DPP4 inhibitors may help us to better understand the effect of treatment frequency on GV in patients with T2DM.

\section{CONCLUSIONS}

Despite the different dosing frequencies, onceweekly trelagliptin and once-daily alogliptin improved glycemic control and reduced GV without inducing hypoglycemia.

\section{ACKNOWLEDGEMENTS}

The authors would like to thank the patients who have participated in this trial and their families.
Funding. Sponsorship for this study, the journal's rapid service fee, and open access fee were funded by Takeda Pharmaceutical Company Ltd. Takeda Pharmaceutical Company Ltd prepared the protocol, handled the clinical research organizations responsible for site management and monitoring, data management, and statistical analysis and was involved in the preparation and publication of the manuscript. All authors had full access to all of the data in this study and take complete responsibility for the integrity of the data and accuracy of the data analysis.

Medical Writing Assistance. Writing assistance from Sabah Farooq of FireKite, an Ashfield company, part of UDG Healthcare plc, was utilized during the development of this manuscript, which was funded by Takeda Pharmaceutical Company Ltd, in compliance with Good Publication Practice 3 ethical guidelines (Battisti et al., Ann Intern Med 2015;163:461-4).

Other Assistance. The authors are also grateful to Michinori Arimitsu, of A2 Healthcare Corporation, for reviewing the study protocol and the clinical study report, for the development of the statistical analysis plan, and for the statistical analysis.

Authorship. All named authors meet the International Committee of Medical Journal Editors (ICMJE) criteria for authorship for this article, take responsibility for the integrity of the work as a whole, and have given their approval for this version to be published.

Authorship Contributions. Rimei Nishimura and Takeshi Osonoi contributed to the study design, interpretation of the data, and critically reviewed and edited the manuscript. TO also contributed toward data acquisition. YK contributed to the study design, data analysis and drafting the manuscript. KM contributed to the interpretation of the data and drafting the manuscript. YS contributed to the study design, data analysis, interpretation of the data, and drafting the manuscript. All authors read and approved the final manuscript and all 
authors agree to be accountable for all aspects of the work, which includes ensuring that questions related to the accuracy or integrity of any part of the work are appropriately investigated and resolved.

Disclosures. Rimei Nishimura has received honoraria from Astellas Pharma Inc, Nippon Boehringer Ingelheim Co. Ltd, Eli Lilly Japan K.K., Kissei Pharmaceutical Co. Ltd, Medtronic Japan Co. Ltd, MSD, Novartis Pharma K.K., Novo Nordisk Pharma Ltd, Sanofi K.K., Sumitomo Dainippon Pharma Co. Ltd., Takeda Pharmaceutical Co., Ltd. He has also received a grant from the Japan Diabetes Foundation. Takeshi Osonoi has received honoraria from Takeda Pharmaceutical Co., Ltd., Novo Nordisk Pharma Ltd, Sanwa Kagaku Kenkyusho Co., Ltd., Astellas Pharma Inc, Mitsubishi Tanabe Pharma Corporation, AbbVie GK, AstraZeneca K.K., Eli Lilly Japan K.K., Daiichi Sankyo Co., Ltd., Bayer Yakuhin, Ltd, Sanofi K.K., Japan Tobacco Inc., Abbott, Poxel SA, Kowa Co., Ltd. Yasuhiro Koike is an employee of Takeda Pharmaceutical Co., Ltd, which sponsored the study. Kouji Miyata is an employee of Takeda Pharmaceutical Co., Ltd, which sponsored the study. Yukio Shimasaki is an employee of Takeda Pharmaceutical Co., Ltd, which sponsored the study.

Compliance with Ethics Guidelines. All procedures performed in studies involving human participants were in accordance with the ethical standards of the institutional and/or national research committee and with the 1964 Helsinki declaration and its later amendments or comparable ethical standards. Informed consent was obtained from all individual participants included in the study. The study protocol, informed consent form, and other regulation-specified documents were reviewed and approved by the Independent Ethics Committees at Nakakinen Clinic.

Data Availability. Takeda makes patientlevel, de-identified data sets and associated documents available after applicable marketing approvals and commercial availability have been received, an opportunity for the primary publication of the research has been allowed, and other criteria have been met as set forth in Takeda's Data Sharing Policy (see https://www. takedaclinicaltrials.com/ for details). To obtain access, researchers must submit a legitimate academic research proposal for adjudication by an independent review panel, who will review the scientific merit of the research and the requestor's qualifications and conflict of interest that can result in potential bias. Once approved, qualified researchers who sign a data sharing agreement are provided access to these data in a secure research environment.

Open Access. This article is distributed under the terms of the Creative Commons Attribution-NonCommercial 4.0 International License (http://creativecommons.org/licenses/ by-nc/4.0/), which permits any noncommercial use, distribution, and reproduction in any medium, provided you give appropriate credit to the original author(s) and the source, provide a link to the Creative Commons license, and indicate if changes were made.

\section{REFERENCES}

1. Cho NH, Shaw JE, Karuranga S, et al. IDF Diabetes Atlas: global estimates of diabetes prevalence for 2017 and projections for 2045. Diabetes Res Clin Pract. 2018;138:271-81.

2. International Diabetes Federation. IDF Diabetes Atlas. 8th ed. Brussels: International Diabetes Federation; 2017.

3. Suh S, Kim JH. Glycemic variability: how do we measure it and why is it important? Diabetes Metab J. 2015;39(4):273-82.

4. Nathan DM, Turgeon H, Regan S. Relationship between glycated haemoglobin levels and mean glucose levels over time. Diabetologia. 2007;50(11):2239-44.

5. Saudek CD, Brick JC. The clinical use of hemoglobin A1c. J Diabetes Sci Technol. 2009;3(4):629-34.

6. Bonds DE, Miller ME, Bergenstal RM, et al. The association between symptomatic, severe hypoglycaemia and mortality in type 2 diabetes: retrospective epidemiological analysis of the ACCORD study. BMJ. 2010;340:b4909. 
7. Hsu PF, Sung SH, Cheng HM, et al. Association of clinical symptomatic hypoglycemia with cardiovascular events and total mortality in type 2 diabetes: a nationwide population-based study. Diabetes Care. 2013;36(4):894-900.

8. Sartore G, Chilelli NC, Burlina S, Lapolla A. Association between glucose variability as assessed by continuous glucose monitoring (CGM) and diabetic retinopathy in type 1 and type 2 diabetes. Acta Diabetol. 2013;50(3):437-42.

9. Su G, Mi S, Tao H, et al. Association of glycemic variability and the presence and severity of coronary artery disease in patients with type 2 diabetes. Cardiovasc Diabetol. 2011;10:19.

10. Smith-Palmer J, Brandle M, Trevisan R, Orsini Federici M, Liabat S, Valentine W. Assessment of the association between glycemic variability and diabetes-related complications in type 1 and type 2 diabetes. Diabetes Res Clin Pract. 2014;105(3):273-84.

11. Zhang Y, Hu G, Yuan Z, Chen L. Glycosylated hemoglobin in relationship to cardiovascular outcomes and death in patients with type 2 diabetes: a systematic review and meta-analysis. PLoS One. 2012;7(8):e42551.

12. Monnier L, Mas E, Ginet C, et al. Activation of oxidative stress by acute glucose fluctuations compared with sustained chronic hyperglycemia in patients with type 2 diabetes. JAMA. 2006;295(14):1681-7.

13. Ceriello A, Esposito K, Piconi L, et al. Glucose "peak" and glucose "spike": impact on endothelial function and oxidative stress. Diabetes Res Clin Pract. 2008;82(2):262-7.

14. Vella A. Mechanism of action of DPP-4 inhibitors-new insights. J Clin Endocrinol Metab. 2012;97(8):2626-8.

15. Gerich J. Pathogenesis and management of postprandial hyperglycemia: role of incretin-based therapies. Int J Gen Med. 2013;6:877-95.

16. Patel BD, Ghate MD. Recent approaches to medicinal chemistry and therapeutic potential of dipeptidyl peptidase-4 (DPP-4) inhibitors. Eur J Med Chem. 2014;74:574-605.

17. Takeda Pharmaceutical Company Ltd. Zafatek ${ }^{\circledR}$ (trelagliptin succinate) tablet prescribing information (Japanese). 2016. https://www.takedamed. com/accept/?url=\%2Fcontent $\% 2$ Fmedicine $\% 2 F p d f$ \%2Fzafatek.pdf. Accessed May 2, 2017.

18. Inagaki $\mathrm{N}$, Onouchi $\mathrm{H}$, Maezawa $\mathrm{H}$, Kuroda $\mathrm{S}$, Kaku K. Once-weekly trelagliptin versus daily alogliptin in Japanese patients with type 2 diabetes: a randomised, double-blind, phase 3, non-inferiority study. Lancet Diabetes Endocrinol. 2015;3(3):191-7.

19. Pharmaceutical and Food Safety Bureau Ministry of Health Labour and Welfare. On release of the Guideline for Clinical Evaluation of Oral Hypoglycemic Agents. 2010. https://www.pmda.go.jp/ files/000208194.pdf. Accessed May 2, 2017.

20. Action to Control Cardiovascular Risk in Diabetes Study Group, Gerstein HC, Miller ME, et al. Effects of intensive glucose lowering in type 2 diabetes. N Engl J Med. 2008;358(24):2545-59.

21. Currie CJ, Peters JR, Tynan A, et al. Survival as a function of $\mathrm{HbA}(1 \mathrm{c})$ in people with type 2 diabetes: a retrospective cohort study. Lancet. 2010;375(9713):481-9.

22. Dandona P. Minimizing glycemic fluctuations in patients with type 2 diabetes: approaches and importance. Diabetes Technol Ther. 2017;19(9): 498-506.

23. Nomoto H, Miyoshi H, Sugawara H, et al. A randomized controlled trial comparing the effects of dapagliflozin and DPP-4 inhibitors on glucose variability and metabolic parameters in patients with type 2 diabetes mellitus on insulin. Diabetol Metab Syndr. 2017;9:54.

24. Park SE, Lee BW, Kim JH, et al. Effect of gemigliptin on glycaemic variability in patients with type 2 diabetes (STABLE study). Diabetes Obes Metab. 2017;19(6):892-6.

25. Takebayashi K, Suzuki T, Naruse R, et al. Long-term effect of alogliptin on glycemic control in Japanese patients with type 2 diabetes: a 3.5-year observational study. J Clin Med Res. 2017;9(9):802-8.

26. Inagaki N, Sano H, Seki Y, Kuroda S, Kaku K. Longterm safety and efficacy of a novel once-weekly oral trelagliptin as monotherapy or in combination with an existing oral antidiabetic drug in patients with type 2 diabetes mellitus: a 52-week open-label, phase 3 study. J Diabetes Investig. 2016;7(5):718-26.

27. Yabe D, Eto T, Shiramoto M, et al. Effects of DPP-4 inhibitor linagliptin and GLP-1 receptor agonist liraglutide on physiological response to hypoglycaemia in Japanese subjects with type 2 diabetes: a randomized, open-label, 2-arm parallel comparative, exploratory trial. Diabetes Obes Metab. 2017;19(3):442-7.

28. Inagaki N, Onouchi H, Sano H, Funao N, Kuroda S, Kaku K. SYR-472, a novel once-weekly dipeptidyl peptidase-4 (DPP-4) inhibitor, in type 2 diabetes 
mellitus: a phase 2, randomised, double-blind, placebo-controlled trial. Lancet Diabetes Endocrinol. 2014;2(2):125-32.

29. Inagaki N, Sano H, Seki Y, Kuroda S, Kaku K. Efficacy and safety of once-weekly oral trelagliptin switched from once-daily dipeptidyl peptidase- 4 inhibitor in patients with type 2 diabetes mellitus: an open-label, phase 3 exploratory study. J Diabetes Investig. 2018;9(2):354-9.

30. Ma D, Yu Y, Yu X, Zhang M, Yang Y. The changes of leukocyte telomere length and telomerase activity after sitagliptin intervention in newly diagnosed type 2 diabetes. Diabetes Metab Res Rev. 2015;31(3):256-61.

31. Dalle S, Burcelin R, Gourdy P. Specific actions of GLP-1 receptor agonists and DPP4 inhibitors for the treatment of pancreatic beta-cell impairments in type 2 diabetes. Cell Signal. 2013;25(2):570-9.

32. Urata H, Eimoto M. Points to note when using CGM. Patient explanation [Japanese]. Calm. 2015;2(1):8-11. 